\title{
Gray Matter Differences Correlate with Spontaneous Strategies in a Human Virtual Navigation Task
}

\author{
Véronique D. Bohbot, ${ }^{1}$ Jason Lerch, ${ }^{2}$ Brook Thorndycraft, ${ }^{1}$ Giuseppe Iaria, ${ }^{3}$ and Alex P. Zijdenbos ${ }^{2,4}$ \\ ${ }^{1}$ Douglas, Department of Psychiatry, McGill University, Verdun, Quebec, Canada H4H 1R3, ${ }^{2}$ Montreal Neurological Institute, McGill University, Montreal, \\ Quebec, Canada H3A 2B4, ${ }^{3}$ Dipartimento di Psicologia, Università di Roma "La Sapienza" and Istituti di Ricovero e Cura a Carattere Scientifico Fondazione \\ Santa Lucia, Rome, Italy, and ${ }^{4}$ Neuralyse, Montreal, Quebec, Canada H4P 2R2
}

Young healthy participants spontaneously use different strategies in a virtual radial maze, an adaptation of a task typically used with rodents. Functional magnetic resonance imaging confirmed previously that people who used spatial memory strategies showed increased activity in the hippocampus, whereas response strategies were associated with activity in the caudate nucleus. Here, voxel based morphometry was used to identify brain regions covarying with the navigational strategies used by individuals. Results showed that spatial learners had significantly more gray matter in the hippocampus and less gray matter in the caudate nucleus compared with response learners. Furthermore, the gray matter in the hippocampus was negatively correlated to the gray matter in the caudate nucleus, suggesting a competitive interaction between these two brain areas. In a second analysis, the gray matter of regions known to be anatomically connected to the hippocampus, such as the amygdala, parahippocampal, perirhinal, entorhinal and orbitofrontal cortices were shown to covary with gray matter in the hippocampus. Because low gray matter in the hippocampus is a risk factor for Alzheimer's disease, these results have important implications for intervention programs that aim at functional recovery in these brain areas. In addition, these data suggest that spatial strategies may provide protective effects against degeneration of the hippocampus that occurs with normal aging.

Key words: spatial memory; hippocampus; caudate nucleus; virtual environment; response learning; basal ganglia

\section{Introduction}

To move adaptively within the environment, humans spontaneously adopt different navigational strategies, which rely on different parts of the brain (Iaria et al., 2003). To reach a target location, one may use a "spatial memory strategy" by learning the relationships between environmental landmarks (stimulusstimulus associations). This strategy is a form of explicit memory based on a cognitive map (O'Keefe and Nadel, 1978) that allows a target to be reached in a direct path from any given direction. This type of flexible navigation has been shown to depend on the hippocampus (Maguire et al., 1998; Bohbot et al., 2004). Alternatively, one can navigate without knowledge of the relationships between environmental landmarks, but instead by using a series of turns at precise decision points or stimuli (e.g., turn left at the corner, then turn right after the park, etc.). The successful repe-

Received Jan. 5, 2007; revised June 7, 2007; accepted June 26, 2007.

This work was supported by Natural Sciences and Engineering Research Council Operating Grant 239920, start-up funds from the Douglas Hospital Research Centre, Canadian Institutes of Health Research Operating Grant 64381, and Fonds de la recherche en santé du Québec fund numbers 3234 and 2828 (V.D.B.). We thank Remi Quirion, Peter Shizgal, Norm White, Ken Bruce, Shumita Roy, Samuel Mckenzie, Nicolas Andersen, and the reviewers for valuable feedback on a previous version of this manuscript.

Correspondence should be addressed to Dr. Véronique D. Bohbot, Department of Psychiatry, McGill University, Douglas, Frank B. Common Building, 6875 Boulevard LaSalle, Verdun, Quebec, Canada H4H 1R3. E-mail: veronique.bohbot@mcgill.ca.

G. Iaria's present address: University of British Columbia, 2550 Willow Street, Vancouver, British Columbia, Canada V5Z3N9.

DOI:10.1523/JNEUROSCI.1763-07.2007

Copyright $\odot 2007$ Society for Neuroscience $\quad$ 0270-6474/07/2710078-06\$15.00/0 tition of this nonspatial strategy leads to a "response strategy" (stimulus-response associations) known to involve the caudate nucleus (Packard et al., 1989; Hartley et al., 2003; Iaria et al., 2003), a form of implicit memory or habit.

In a previous study conducted in our laboratory (Iaria et al., 2003), 50 young healthy participants performed a virtual navigation task on a computer monitor, the "virtual maze task," which could be solved by using either of these two strategies. The participants had to learn the locations of objects hidden at the end of paths extending from a radial maze. A probe trial that involved the removal of all landmarks was used to identify the participants adopting a spatial strategy because we predicted that only this group would show an increase in errors. Based on self report and the probe trial, we found that about one-half of the participants spontaneously used the response strategy. They made fewer errors on the probe trial and reported making a series of displacements (e.g., take the second arm to the left, then take the next left) from a given starting point or stimulus. The other half spontaneously used spatial memory. They made significantly more errors on the probe trial and reported learning the locations of target objects in relation to multiple landmarks. These proportions matched those observed previously in rodents (McDonald and White, 1994). In that particular task, the response strategy was more efficient than the spatial memory strategy, as evidenced by fewer errors and less time to complete the task. With additional training, $40 \%$ of the people using the spatial memory strategy shifted to the more efficient response strategy, as has been dem- 


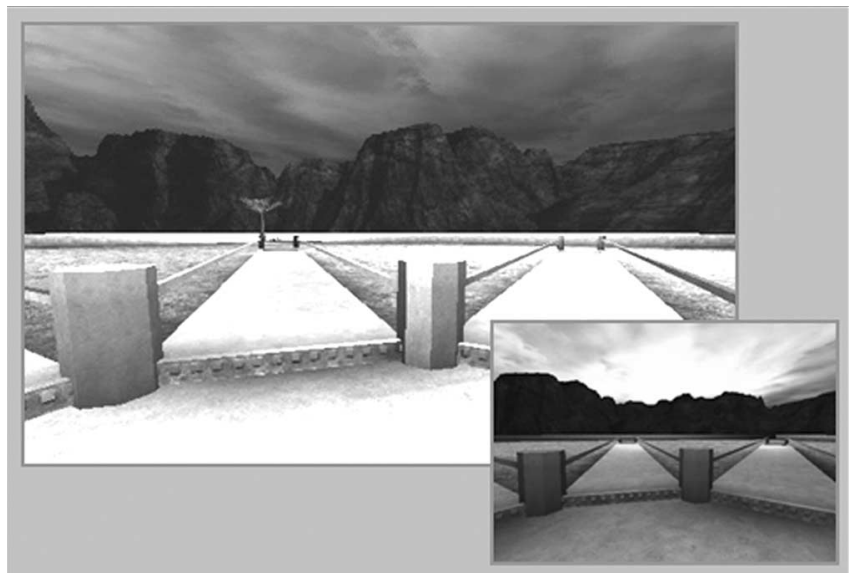

Figure 1. Two views of the virtual environment used in this study. Note the tree and mountains that are part of the landscape in one view and the sunset in the other. Another tree and large mountain were present in the virtual environment.

onstrated in rats (Packard and McGaugh, 1996). A functional magnetic resonance imaging (fMRI) study showed that the hippocampus was significantly activated only in spatial learners, whereas the caudate nucleus showed significant sustained activity in response learners (Iaria et al., 2003). In this experiment, we sought to investigate whether the spontaneous use of a given strategy covaried with morphological differences in the hippocampus or caudate nucleus of the human brain with three voxel-based morphometry (VBM) analyses.

\section{Materials and Methods}

Participants. The MRI scans of 30 young, healthy participants (17 men and 13 women, with a mean age of $27.9 \pm 4.1$ years), with no particular expertise and who had been tested previously in our laboratory, were used in this study (Iaria et al., 2003).

Behavioral task. Participants were tested using a virtual environment representing an eight-arm radial maze. The environment was designed by the researchers, using a commercial computer game editor (Unreal; Epic Games, Raleigh, NC). It was displayed to participants sitting at a desk, $\sim 20$ inches from a standard 17 inch computer screen. The eight arms originated in a central platform, and the maze was surrounded by mountains, sky, a sunset, two trees, and a short wall separating the trees from the mountains (Fig. 1). Each of the eight arms terminated in a staircase leading to a lowered chamber. Only four of these had objects (square boxes) in them. Participants were unable to see the objects from the center; therefore, they had to remember which arms contained objects and which did not to perform the task without errors. The purpose of this test was to determine which strategies participants used to remember the locations of objects in an environment. A participant could use a spatial memory strategy by using two or more landmarks available in the environment and the spatial relationships between them. However, one could use a nonspatial strategy by remembering a sequence of left and right turns, or numbering or naming the eight arms from a single starting position without considering the elements present in the environment. To move within the environment, participants were asked to use only the forward, left, and right arrow keys on a keyboard. The use of a keyboard to navigate in the virtual environment was favored over the joystick to minimize convoluted paths or entries into undesired arms by people without previous gaming experience. With a keyboard, people only press the forward button when they face the arm they wish to enter. Still, we asked participants to tell us whether they had previous experience with video games. We rated their answer as 1 for yes, 2 for some, and 3 for none, and found no correlation with errors on the probe trial $(r=-0.27$, not significant). Furthermore, before starting the task, participants had the opportunity to move around in a different virtual room, to become accustomed to navigating using only the arrow keys.
Once participants were comfortable moving around in the environment, the task began. The experiment included three types of trials (A, B, and $C$ ). In each trial, participants started in the center and always faced the same direction. In the first part of all trials, four of the eight arms were blocked. Participants were asked to retrieve the objects in the open arms and remember where they had been to avoid those arms in the next part of the trial. In the second part of every trial, all eight arms were open, but only those four that had not already been visited contained objects. The choice of an unrewarded path counted as an error. In trial A, arms 1, 3, 4, and 6 were open in the first part, meaning that in the second part the objects were located in arms 2, 5, 7, and 8. In trial B, arms 2, 3, 7, and 8 were open in the first part, and participants had to choose 1, 4, 5, and 6 in the second part to perform without error. The first part of trial $\mathrm{C}$ (the probe trial) was identical to the first part of trial A; however, in the second part of this trial, the trees were removed and the walls were raised between the maze and the landscape to conceal the mountains and sunset. Participants who used the landmarks in a spatial memory strategy were expected to make errors during the probe trial, whereas those who had used the response strategy based on the starting position could perform well. In each trial, participants were stopped after they retrieved four objects. Participants were given a total of 18 trials. Section 1 consisted of four trials (A, B, A, and C). Sections 2 and 3 together consisted of 10 trials of type A (A, A, A. . .), and Section 4 was identical to Section 1 . At the end of the experiment, there was a debriefing during which the participants were asked to report how they had solved the task from the beginning to the end of the experiment. Although the original test design included four test sections and a debriefing, only data from the probe trial $\mathrm{C}$ of the first section were used for the VBM analysis.

MRI acquisition. Participants were scanned at the Montreal Neurological Institute (MNI) using a Siemens (New York, NY) Sonata 1.5-tesla superconducting magnet system with the International Consortium for Brain Mapping protocol (Mazziotta et al., 1995). This protocol generates T1-weighted image volumes with a $1 \mathrm{~mm}$ isotropic resolution using a three-dimensional spoiled gradient echo acquisition with sagittal volume excitation (repetition time, 22; echo time, 10; flip angle, $30^{\circ}$; $1401 \mathrm{~mm}$ sagittal slices). The rectangular field of view for the sagittal images was $256 \mathrm{~mm}$ (SI) by $224 \mathrm{~mm}$ (AP).

Voxel-based morphometry. Voxel-based morphometry is a computational approach to neuroanatomy that measures differences in the local density of brain tissue through a voxelwise comparison of multiple brain images (Ashburner and Friston, 2000) throughout the brain. MRI scans were spatially normalized by linear transformation into a stereotaxic coordinate system based on the Talairach atlas (Talairach and Tournoux, 1988; Collins et al., 1994). They were corrected for intensity nonuniformity (shading artifact) using the N3 software package (Sled et al., 1998). Each voxel was automatically labeled as white matter, gray matter, CSF, or background using INSECT (intensity normalized stereotaxic environment for the classification of tissues), a method relying on an artificial neural network classifier (Zijdenbos et al., 2002). The skull and dura were masked from the brain. Gray matter was used for the VBM analyses to investigate whether there were differences between spatial and response learners in the hippocampus and caudate nucleus. The gray matter was smoothed using an $8 \mathrm{~mm}$ full-width at half-maximum Gaussian kernel, which presents a weighted average of the amount of gray matter in the area surrounding the voxel, thereby measuring regional gray-matter density rather than the density of each voxel. Generalized linear models were used to determine whether gray matter correlated with the number of errors in the probe trial (Worsley et al., 1992). We selected these variables based on our previous fMRI study (Iaria et al., 2003) where we found that the strategies evaluated with verbal reports or probe trials were critical to produce the functional dissociation between activity in the hippocampus and caudate nucleus. Consequently, in the current study, we hypothesized that these measures of navigational strategies would be sensitive to gray matter changes. Specifically, a first VBM analysis was performed to study the relationship between human brain morphology and the spontaneous bias in adopting navigational strategies. A comparison was made between the gray-matter density obtained from the MRIs of all participants and the errors made on the probe trial, which provide an objective measure of navigational strategy. It was expected that participants who 

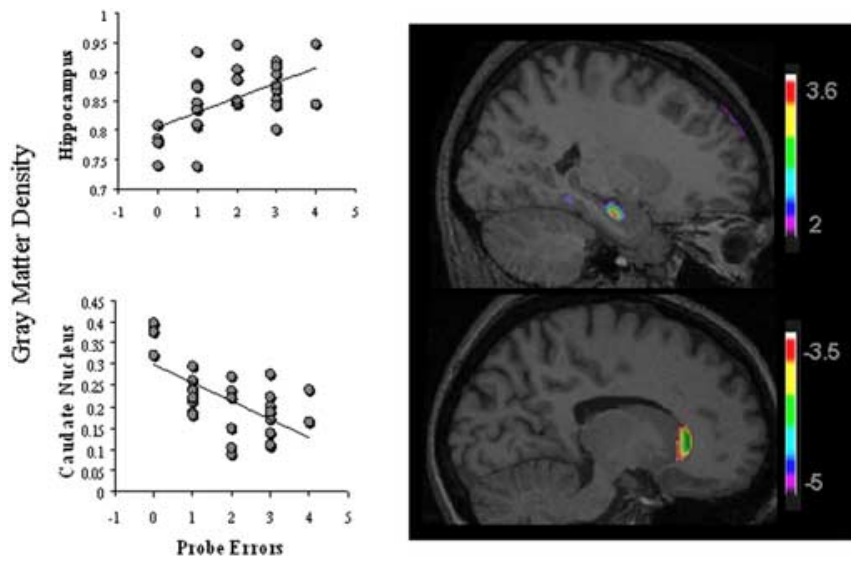

Figure 2. The diagrams show the regression analyses between the gray-matter density (hippocampus and caudate nucleus) and the errors made by the entire group of participants while performing the probe trial. The right side of the figure shows the results superimposed onto an anatomical MRI and displayed in the sagittal plane. Gray-matter density in the right hippocampus at the peak ( $x=24, y=-13, z=-20 ; t=3.55$ ) was correlated with spatial memory strategies ( $r=0.56 ; p<0.005$; top), whereas the density in the head of the caudate nucleus at the peak ( $x=-14, y=28, z=4 ; t=-4.33$ ) was correlated with response learning strategies ( $r=-0.63 ; p<0.005$; bottom). Other than a negative correlation between probe errors and the tail of the caudate nucleus $(x=-26, y=-32, z=3 ; t=-4.07$; correlation coefficient $r=0.56 ; p<0.005)$, no other region of the brain crossed the threshold for significance corrected for multiple comparisons. The color bars illustrate the range of $t$ statistical values.

used the relationships between environmental landmarks (i.e., the spatial strategy) would make errors when trying to find the objects during the probe trial, whereas participants who ignored the landmarks and navigated by response strategies would perform well. Consequently, higher numbers of errors on the probe trial show an indication that the participants relied on landmarks with the spatial strategy. The results of the first VBM analysis were used to regress the gray-matter value at the peak voxel (MNI space coordinates, $x=24, y=-13, z=-20$ ) in the hippocampus against the entire MRI volume in all participants. For example, the gray matter value of a spatial learner at this coordinate was 0.88 whereas the gray matter value of a response learner at this coordinate was 0.76. These values were regressed against the entire MRI volume in each participant. This regression tests the hypothesis that the gray matter in the network of regions known to be anatomically linked to the hippocampus covaried with hippocampal gray matter. This method was validated previously with cortical thickness maps (Lerch et al., 2006; He et al., 2007).

The output of the analysis was displayed as a statistical map, overlaid on an image of an MRI scan, which showed regions of significant difference in gray matter between the groups under comparison. The $t$ statistic threshold for the hippocampus and caudate nucleus, based on the number of test participants $(n=30)$, was calculated using random field theory to be 3.40 at $p<0.001$ and 4.25 at $p<0.0001$, uncorrected for multiple comparisons, because of our a priori hypotheses concerning these structures. For the entire volume, it was calculated to be 6.0, corrected for multiple comparisons at $p<0.05$. Note that the highest significance level reported for correlation was $p<0.005$ for a critical value of $0.46(\mathrm{df}=29)$ and $p<0.005$ for a critical value of $0.58(\mathrm{df}=17)$; however, the actual significance level that was reached was much higher.

\section{Results}

Using the behavioral data of the 30 participants, a comparison was made between gray-matter density, which is the relative proportion of gray matter in a given volume of tissue, and errors made on the probe trial. This analysis generated a positive peak in the right hippocampus $(x=24, y=-13, z=-20 ; t$ statistic $=$ 3.55) (Fig. 2). The correlation coefficient between the graymatter density at the peak in hippocampus and the number of

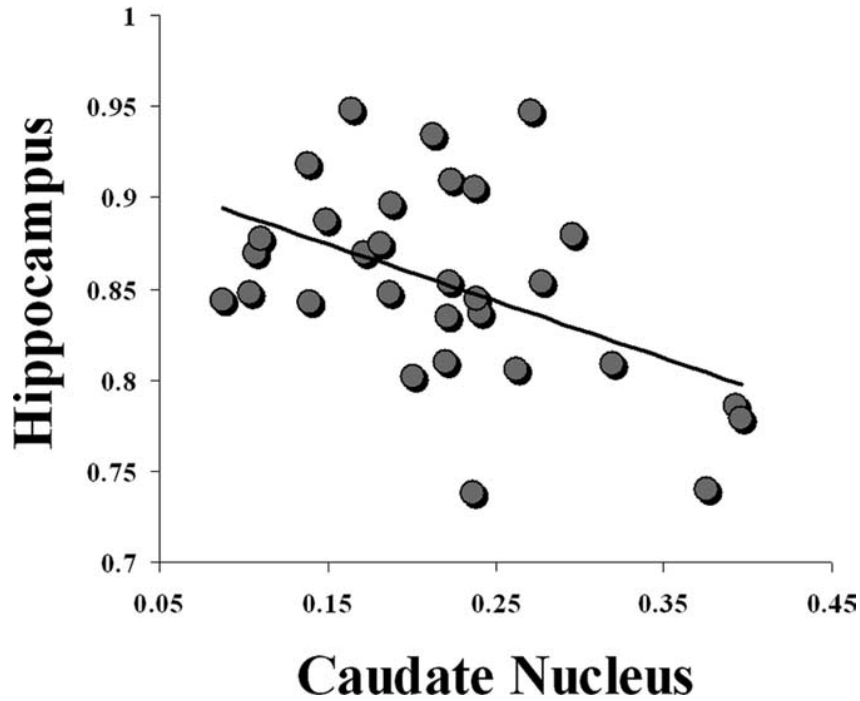

Figure 3. Inverse correlation $(r=-0.43 ; p<0.05)$ between gray-matter density in hippocampus $(x=24, y=-13, z=-20)$ and the head of the caudate nucleus $(x=-14, y=$ $28, z=4$ ) at the peaks resulting from the first VBM analysis in all 30 participants (Fig. 2).

probe errors was $r=0.56(p<0.005)$. For subjects relying on a spatial strategy, the more errors made when landmarks were removed, the higher the gray-matter density in the hippocampus. In addition, a negative peak was found in the head $(x=-14, y=$ $28, z=4 ; t$ statistic $=-4.33$; correlation coefficient $r=-0.63$; $p<0.005)$ (Fig. 2) and tail $(x=-26, y=-32, z=3 ; t=-4.07$; correlation coefficient $r=0.56 ; p<0.005)$ of the left caudate nucleus. The more people rely on spatial memory, the lower the gray-matter density in the left caudate nucleus. Thus, people who did not use multiple environmental landmarks made fewer errors on the probe trials and had higher gray-matter density in the left caudate nucleus. Interestingly, the gray-matter densities in the caudate nucleus and hippocampus that resulted from the first VBM analysis were inversely correlated $(r=-0.43 ; p<0.05)$ (Fig. 3).

In the second VBM analysis, a network of regions known to be anatomically linked to the hippocampus showed increases in gray matter that covaried with the gray matter at the peak voxel in the right hippocampus. Specifically, as the gray matter in the hippocampus was higher, so was the gray matter in the contralateral hippocampus, the amygdala, and the parahippocampal, perirhinal, entorhinal, and orbitofrontal cortices bilaterally (Fig. 4). In other words, the participants with the highest amounts of gray matter in the hippocampus, at the coordinates sensitive to our navigation paradigm, also had the highest amounts of gray matter in the above-mentioned network of neuroanatomically connected regions. This also means that the participants with the lowest amounts of gray matter in the hippocampus also had the lowest amounts of gray matter in the above-mentioned network.

\section{Discussion}

The spontaneous strategies measured in the present experiment may be representative of strategies used in everyday life. The probability that an individual uses a spatial strategy is higher when the gray-matter density in the hippocampus is high. Conversely, the probability that an individual navigates with the response strategy is higher with high gray-matter density in the caudate nucleus. The most efficient navigational strategy will vary with situational demands. For example, when the start and target locations are consistently in the same location, such as when 


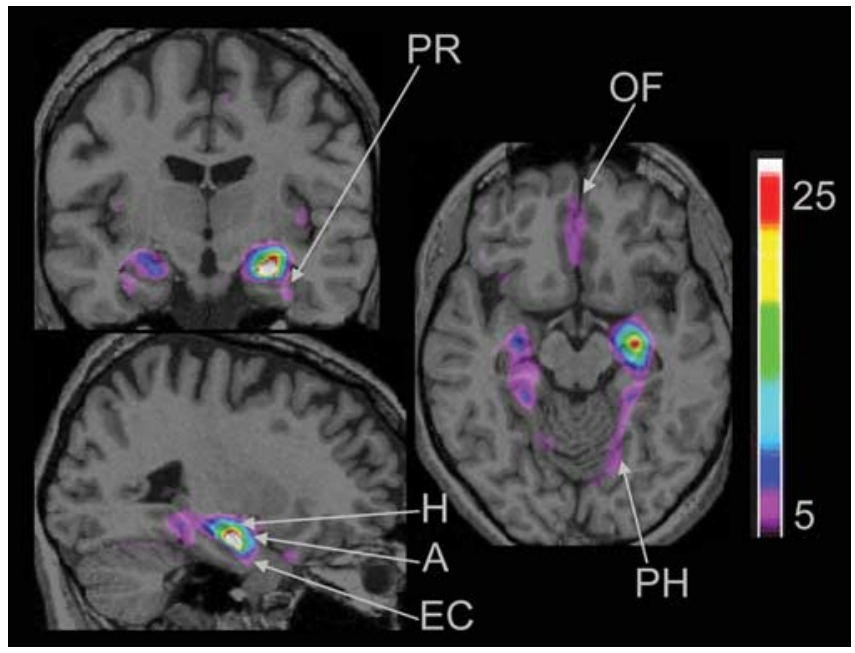

Figure 4. Regions of the brain that covaried with hippocampal gray matter extracted at the coordinates resulting from the first VBM analysis $(x=24, y=-13, z=-20)$. The coronal section (top left) is displayed at the coordinate $y=-14$, the sagittal section is displayed at $x=$ 25 , and the axial section is displayed at $z=-14$. A, Amygdala; $H$, hippocampus; $E C$, entorhinal cortex; PR, perirhinal cortex; $\mathrm{PH}$, parahippocampal cortex; $0 \mathrm{~F}$, orbitofrontal cortex. The color bar illustrates the range of $t$ statistical values.

going to the same workplace from home everyday, the repeated use of a particular route with the response strategy is more efficient than the spatial strategy, because less time is spent deciding which way to go and wrong turns can be avoided. However, learning the spatial relationship among environmental landmarks with the spatial strategy can be beneficial because it allows one to derive shortcuts. For example, one may know that the store is west of home and the workplace is south of home. Therefore, when going to the store directly from work, one must head northwest. Not having learned the relationship between the store and the workplace, the person who was previously using a response strategy to travel to the store and to work from home would be unable to take such a shortcut and would get lost or would have to take a detour home when going to the store from work. In the current experiment, where the start and target locations remained constant, the response strategy was more efficient in terms of latencies and errors. In a virtual town that requires reaching a target from any direction, the spatial strategy is more efficient because it allows deriving shortcuts. Consequently, the most efficient navigators are likely to be those that are flexible at using spatial or response strategies on demand (Hartley et al., 2003).

The data also suggest that there is a competitive interaction between the hippocampus and caudate nucleus, one or the other being optimal for different tasks. The higher probability of using one strategy or the other may underlie the negative correlation in gray-matter density reported here. Consistent with our findings, Poldrack et al. (2001) reported that fMRI activity in the caudate nucleus was negatively correlated to that of the medial temporal lobe. In a positron emission tomography study, Dagher showed that patients with Parkinson's disease had increased hippocampal activation in a task that normally involved the caudate nucleus in controls (Dagher et al., 2001). Similarly, an increase in hippocampal fMRI activation was measured in patients with early stage Huntington's disease, which was accompanied by attenuated activity in the caudate nucleus during a route recognition task (Voermans et al., 2004). Together, these studies provide evidence for a competitive or cooperative interaction between the hippocampus and caudate nucleus memory systems, depending on whether or not the behavioral strategies subserved by these systems are placed in conflict (Packard et al., 1989; Knowlton et al., 1996).

The brain-behavior correlation implicating spatial and response strategies was also reported in rodents $(\mathrm{McD}$ onald and White, 1994). The number of NMDA receptors that mediate cellular plasticity in the hippocampus correlated with spatial learning in the water maze (Adams et al., 2001). Further, phosphorylated cAMP response element-binding protein, thought to be involved in the formation of long term memory, persisted in the hippocampus only in spatial learners, and in the striatum (caudate nucleus/putamen) only in response learners trained on a cross maze (Colombo et al., 2003). Furthermore, basal levels of acetylcholine in the rat were higher in hippocampus of spatial learners relative to response learners, whereas these levels were higher in the striatum of response learners (McIntyre et al., 2003). Correspondingly, higher hippocampal acetylcholine levels, induced by estrogen administration in ovariectomized female rats, lead to an increase in the proportion of spatial learners (Korol and Kolo, 2002; Marriott and Korol, 2003). Thus, similar to the morphological differences reported in our study, neurobiological differences between spatial and response learners in rodents have been observed in the hippocampus and striatum/caudate nucleus respectively.

In humans, years of expertise was positively correlated with the volume of the posterior hippocampus in taxi drivers (Maguire et al., 2000, 2006). Although in our study the peak showing increased gray matter in the right hippocampus of spatial learners was located in the anterior segment, it overlapped with the expanded region of hippocampus reported in taxi drivers, but not with the atrophied segment, located at the boundary with the amygdala. Interestingly, a VBM study of way-finding ability (Maguire et al., 2003) showed no correlation between navigation ability and gray-matter density in the hippocampus of unspecialized volunteers. This is consistent with our findings in the light of an fMRI study where correlations with performance on the virtual town showed that unspecialized volunteers who are good navigators activated the hippocampus during wayfinding and the caudate nucleus during route following (Hartley et al., 2003). This provides evidence for the fact that good navigators are flexible at using strategies dependent on their hippocampus or caudate nucleus and may have average gray matter in the hippocampus (Etchamendy and Bohbot, 2007), unlike the spatial learners in the current study who spontaneously use a spatial memory strategy and persist in using that strategy, despite the fact that it was not the most efficient one (Iaria et al., 2003). In light of the taxi driver studies, the increase in gray matter in the hippocampus of spatial learners in our study may be an effect of experience.

Volumetric studies in birds have shown that the size of the avian hippocampus correlates with food-storing behavior, which places a demand on spatial memory, regardless of gender (Clayton, 1995). Changes in volumes were attributed to an increase in cell number, but not neuronal size (Clayton, 1995). Interestingly, controls that were prevented from getting food storing experience were found to have a higher proportion of apoptotic cells (i.e., programmed cell death) relative to birds that experienced storing food (Clayton, 1995). In addition, studies of rodents and birds exposed to an enriched environment have shown increases in the density of neurons, size of cell bodies, dendritic branching, spine density, and levels of nerve growth factor (Greenough et al., 1973; Pham et al., 1999; Pinaud et al., 2002; Kolb et al., 2003). It has been shown that the primate hippocampus generates new 
neurons into adulthood (Kornack and Rakic, 1999) and that learning promotes their survival (Gould et al., 1999), although this may not explain fully the gray matter differences observed in the set of regions anatomically connected to the hippocampus in the current study. In sum, experience with spatial memory is associated with gray matter increases in the hippocampus in a number of species.

Other lines of research have shown that genetic differences contribute to the functioning of the hippocampus (Hariri et al., 2003). Specifically, humans with a BDNF genotype with val homozygote amino acid on codon 66 ( $\mathrm{val}^{66} \mathrm{val}$ ) showed greater hippocampal activity during encoding and recall of novel complex scenes when assessed with fMRI, relative to participants with an amino acid substitution on the same gene (val ${ }^{66} \mathrm{met}$ ), suggesting basic effects of BDNF signaling on hippocampal function (Hariri et al., 2003). Consistent with these results, decreased hippocampal volume was found in carriers of the BDNF met allele relative to val homozygotes (Bueller et al., 2006). Although genes may predispose an individual to structural differences in the hippocampus and caudate nucleus, experience with a particular strategy may be critical to reinforce these differences (Maguire et al., 2000). The current experiment showing that gray matter in the hippocampus was correlated to the gray matter in the amygdala, in the parahippocampal, perirhinal, entorhinal, and orbitofrontal cortices, known to be anatomically connected to the hippocampus and to each other (Suzuki and Amaral, 1990, 1994; Stefanacci et al., 1996), suggested that the gray matter increase observed in the hippocampus of spatial learners may be the result of coactivity of these brain structures during navigation.

The correspondence between spontaneous strategies and gray-matter density presented in the current study has implications for clinical intervention programs that aim at recovering function in patients with dysfunctional hippocampus or caudate nucleus. For example, patients with brain damage to the hippocampus previously tested in this paradigm persisted in using spatial memory despite the fact that they were severely impaired relative to similar patients who successfully used the response strategy (Bohbot et al., 2004). These patients would benefit from intervention programs that help them shift to the use of their intact memory system (Glisky, 1992). Patients with mild cognitive impairment or Alzheimer's disease could benefit from similar memory intervention programs. Moreover, the use of spatial strategies may provide protective effects against normal degeneration of the hippocampus later in life. The reported correspondence between navigational strategies and brain morphology in young healthy participants sheds new light on the relationship between structure and function in human navigational and memory systems.

\section{References}

Adams MM, Smith TD, Moga D, Gallagher M, Wang Y, Wolfe BB, Rapp PR, Morrison JH (2001) Hippocampal dependent learning ability correlates with $N$-methyl-D-aspartate (NMDA) receptor levels in CA3 neurons of young and aged rats. J Comp Neurol 432:230-243.

Ashburner J, Friston KJ (2000) Voxel-based morphometry-the methods. NeuroImage 11:805-821.

Bohbot VD, Iaria G, Petrides M (2004) Hippocampal function and spatial memory: evidence from functional neuroimaging in healthy participants and performance of patients with medial temporal lobe resections. Neuropsychology 18:418-425.

Bueller JA, Aftab M, Sen S, Gomez-Hassan D, Burmeister M, Zubieta JK (2006) BDNF Val66Met allele is associated with reduced hippocampal volume in healthy subjects. Biol Psychiatry 59:812-815.

Clayton NS (1995) The neuroethological development of food-storing memory: a case of use it, or lose it! Behav Brain Res 70:95-102.
Collins DL, Neelin P, Peters TM, Evans AC (1994) Automatic 3D intersubject registration of MR volumetric data in standardized Talairach space. J Comp Assist Tomogr 18:192-205.

Colombo PJ, Brightwell JJ, Countryman RA (2003) Cognitive strategyspecific increases in phosphorylated cAMP response element-binding protein and c-Fos in the hippocampus and dorsal striatum. J Neurosci 23:3547-3554.

Dagher A, Owen AM, Boecker H, Brooks DJ (2001) The role of the striatum and hippocampus in planning: a PET activation study in Parkinson's disease. Brain 124:1020-1032.

Etchamendy N, Bohbot VD (2007) Spontaneous navigational strategies and performance in the virtual town. Hippocampus 17:595-599.

Glisky EL (1992) Acquisition and transfer of declarative and procedural knowledge by memory-impaired patients: a computer data-entry task. Neuropsychologia 30:899-910.

Gould E, Beylin A, Tanapat P, Reeves A, Shors TJ (1999) Learning enhances adult neurogenesis in the hippocampal formation. Nat Neurosci 2:260-265.

Greenough WT, Volkmar FR, Juraska JM (1973) Effects of rearing complexity on dendritic branching in frontolateral and temporal cortex of the rat. Exp Neurol 41:371-378.

Hariri AR, Goldberg TE, Mattay VS, Kolachana BS, Callicott JH, Egan MF, Weinberger DR (2003) Brain-derived neurotrophic factor val66met polymorphism affects human memory-related hippocampal activity and predicts memory performance. J Neurosci 23:6690-6694.

Hartley T, Maguire EA, Spiers HJ, Burgess N (2003) The well-worn route and the path less traveled: distinct neural bases of route following and wayfinding in humans. Neuron 37:877-888.

He Y, Chen ZJ, Evans AC (2007) Small-world anatomical networks in the human brain revealed by cortical thickness from MRI. Cereb Cortex, in press.

Iaria G, Petrides M, Dagher A, Pike B, Bohbot VD (2003) Cognitive strategies dependent on the hippocampus and caudate nucleus in human navigation: variability and change with practice. J Neurosci 23:5945-5952.

Knowlton BJ, Mangels JA, Squire LR (1996) A neostriatal habit learning system in humans. Science 273:1399-1402.

Kolb B, Gorny G, Soderpalm AH, Robinson TE (2003) Environmental complexity has different effects on the structure of neurons in the prefrontal cortex versus the parietal cortex or nucleus accumbens. Synapse 48:149-153.

Kornack DR, Rakic P (1999) Continuation of neurogenesis in the hippocampus of the adult macaque monkey. Proc Natl Acad Sci USA 96:5768-5773.

Korol DL, Kolo LL (2002) Estrogen-induced changes in place and response learning in young adult female rats. Behav Neurosci 116:411-420.

Lerch JP, Worsley K, Shaw WP, Greenstein DK, Lenroot RK, Giedd J, Evans AC (2006) Mapping anatomical correlations across cerebral cortex (MACACC) using cortical thickness from MRI. NeuroImage 31:993-1003.

Maguire EA, Burgess N, Donnett JG, Frackowiak RS, Frith CD, O'Keefe J (1998) Knowing where and getting there: a human navigation network. Science 280:921-924.

Maguire EA, Gadian DG, Johnsrude IS, Good CD, Ashburner J, Frackowiak RS, Frith CD (2000) Navigation-related structural change in the hippocampi of taxi drivers. Proc Natl Acad Sci USA 97:4398-4403.

Maguire EA, Spiers HJ, Good CD, Hartley T, Frackowiak RS, Burgess N (2003) Navigation expertise and the human hippocampus: a structural brain imaging analysis. Hippocampus 13:250-259.

Maguire EA, Woollett K, Spiers HJ (2006) London taxi drivers and bus drivers: a structural MRI and neuropsychological analysis. Hippocampus 16:1091-1101.

Marriott LK, Korol DL (2003) Short-term estrogen treatment in ovariectomized rats augments hippocampal acetylcholine release during place learning. Neurobiol Learn Mem 80:315-322.

Mazziotta JC, Toga AW, Evans A, Fox P, Lancaster J (1995) A probabilistic atlas of the human brain: theory and rationale for its development. The International Consortium for Brain Mapping (ICBM). NeuroImage 2:89-101.

McDonald RJ, White NM (1994) Parallel information processing in the water maze: evidence for independent memory systems involving dorsal striatum and hippocampus. Behav Neural Biol 61:260-270. 
McIntyre CK, Marriott LK, Gold PE (2003) Patterns of brain acetylcholine release predict individual differences in preferred learning strategies in rats. Neurobiol Learn Mem 79:177-183.

O'Keefe J, Nadel L (1978) The hippocampus as a cognitive map. Oxford: Clarendon.

Packard MG, McGaugh JL (1996) Inactivation of hippocampus or caudate nucleus with lidocaine differentially affects expression of place and response learning. Neurobiol Learn Mem 65:65-72.

Packard MG, Hirsh R, White NM (1989) Differential effects of fornix and caudate nucleus lesions on two radial maze tasks: evidence for multiple memory systems. J Neurosci 9:1465-1472.

Pham TM, Soderstrom S, Winblad B, Mohammed AH (1999) Effects of environmental enrichment on cognitive function and hippocampal NGF in the non-handled rats. Behav Brain Res 103:63-70.

Pinaud R, Tremere LA, Penner MR, Hess FF, Robertson HA, Currie RW (2002) Complexity of sensory environment drives the expression of candidate-plasticity gene, nerve growth factor induced-A. Neuroscience 112:573-582.

Poldrack RA, Clark J, Pare-Blagoev EJ, Shohamy D, Creso Moyano J, Myers C, Gluck MA (2001) Interactive memory systems in the human brain. Nature 414:546-550.

Sled JG, Zijdenbos AP, Evans AC (1998) A nonparametric method for au- tomatic correction of intensity nonuniformity in MRI data. IEEE Trans Med Imaging 17:87-97.

Stefanacci L, Suzuki WA, Amaral DG (1996) Organization of connections between the amygdaloid complex and the perirhinal and parahippocampal cortices in macaque monkeys. J Comp Neurol 375:552-582.

Suzuki WA, Amaral DG (1990) Cortical inputs to the CAl field of the monkey hippocampus originate from the perirhinal and parahippocampal cortex but not from area TE. Neurosci Lett 115:43-48.

Suzuki WA, Amaral DG (1994) Perirhinal and parahippocampal cortices of the macaque monkey: cortical afferents. J Comp Neurol 350:497-533.

Talairach J, Tournoux P (1988) Co-planar stereotaxic atlas of the human brain: 3-dimensional proportional system-an approach to cerebral imaging. New York: Thieme Medical.

Voermans NC, Petersson KM, Daudey L, Weber B, van Spaendonck KP, Kremer HP, Fernandez G (2004) Interaction between the human hippocampus and the caudate nucleus during route recognition. Neuron 43:427-435.

Worsley KJ, Evans AC, Marrett S, Neelin P (1992) A three-dimensional statistical analysis for CBF activation studies in human brain. J Cereb Blood Flow Metab 12:900-918.

Zijdenbos AP, Forghani R, Evans AC (2002) Automatic "pipeline" analysis of 3-D MRI data for clinical trials: application to multiple sclerosis. IEEE Trans Med Imaging 21:1280-1291. 